\title{
Subjective random number generation and attention deployment during acquisition and overlearning of a motor skill
}

\author{
FREDERICK J. EVANS \\ Carrier Foundation, Belle Mead, New Jersey 08502 \\ and CMDNJ-Rutgers Medical School, New Brunswick, New Jersey 08903 \\ and \\ CHARLES GRAHAM \\ Biobehavioral Sciences Laboratory, Midwest Research Institute, Kansas City, Missouri 64110
}

\begin{abstract}
The hypothesis that task attentional requirements vary inversely with increases in the efficiency of task performance was evaluated. Three experimental groups $(\mathrm{N}=8, \mathrm{~N}=6, \mathrm{~N}=3)$ were required to learn a two-hand coordination task (THC). To assess changing attentional demands at different levels of skilled THC performance, subjects simultaneously performed a random number generation task (RNG) on two or four trials of the THC task; these trials occurred during initial acquisition, during semiskilled performance, at performance asymptote, or after overlearning. The RNG index showed marked deterioration during initial acquisition ( $p<.005)$, remained below baseline but improved during semiskilled performance $(p<.05)$, and partially and completely recovered to original baseline levels during the mastery and overlearning trials. The RNG procedure may provide a brief, sensitive, and consistent measure of attention deployment during the performance of tasks and in the learning and acquisition of complex skills.
\end{abstract}

Performing two different tasks simultaneously is a common research strategy in studies of human attention. Typically, as attention is concentrated on the primary task and withdrawn from the secondary, or index, task, performance on the index task is impaired (Noble, Trumbo, \& Fowler, 1967). The extent of index task impairment is thought to reflect the difficulty of, or amount of attentive effort required to perform, the primary task. This strategy has been used in several experimental contexts, and the findings have provided substantial support for considering man to be limited in the amount of information he can process per unit time (e.g., Broadbent, 1958; Treisman, 1969). Other workers

This research was supported in part by Contract DADA1771-C-1120 from the U.S. Army Medical Research and Development Command, by Grant MH 19156-07 from the National Institute of Mental Health, Public Health Service, and by the Institute for Experimental Psychiatry, the Institute of Pennsylvania Hospital. It was carried out at the Unit for Experimental Psychiatry. The preparation of the report was supported in part by the Carrier Foundation Division of Research, Belle Mead, New Jersey 08502 (Frederick J. Evans) and in part by the Midwest Research Institute, Kansas City, Missouri 64110 (Charles Graham). We wish to thank H. D. Cohen, M. R. Cook, J. F. Kihlstrom, R. L. Horne, B. E. Lawrence, P. A. Markowsky, E. C. Orne, M. T. Orne, H. M. Pettinati, W. M. Waid, and S. K. Wilson for their many helpful comments. We particularly wish to thank Maribeth A. Miller for her assistance in conducting the experiment, D. Berdan, C. Bendon, and N. Bauer for their help in the data analysis, and D. A. Paskewitz and R. L. Hufgard for their computer skills. have emphasized that the potential contribution of learning in relation to attentional theory needs to be assessed (Kahneman, 1973; LaBerge, 1975; Moray, 1969). Much of the experimental evidence on selective attention has been derived from measures obtained during relatively unpracticed episodes of dual-task performance. Task learning, however, may reduce the high initial demands on attention deployment and with further training enable the individual to maintain superior performance with relatively little attentional effort. The effects of learning may be particularly evident when training is continued past the point of mastery and individuals are required to overlearn a task.

An unresolved problem in evaluating the effects of learning on selective attention has been to develop behavioral index tasks of sufficient sensitivity to reflect fluctuations in attentive effort, not only during singletrial performance, but also during the process of skill acquisition and overlearning. Ideally, such tasks should be (1) sensitive to short-term changes in attention, (2) brief, so that boredom and monotony do not interfere with the process being measured, and (3) relatively unaffected by practice, in order to provide a stable index of attention deployment during repeated dualtask administration. The random number generation task (RNG) provides a behavioral index that appears to meet these criteria. Detailed administration and scoring procedures have been fully described elsewhere (Evans, 1978; Graham \& Evans, 1977). Subjects are asked to 
produce the numbers $1-10$ at random, in time with a metronome (1 beat/sec). A statistical measure of subjective randomness (or sequential response bias) is calculated for the first 100 numbers using an adaptation of Tulving's (1962) subjective organization measure of free recall clustering. This index reflects the frequency with which any number follows any other number compared with chance expectations, modified by a marginal frequency adjustment based on the frequency of use of each of the 10 digits in a response series of the first 100 numbers produced. It is more sensitive to changes in subjective randomness than existing measures in that it provides valuable information about relatively short series of numbers, a major problem in past work with randomization (Wagenaar, 1972). The index (which ranges from .0 to 1.0 ) is reliable and not particularly susceptible to practice or sequence effects. For example, mean RNG scores obtained over three successive trials were $.292, .291$, and $.294(\mathrm{SD}=.045, \mathrm{~N}=12)$. Thus, the RNG task has the potential advantages of being sensitive, brief, and consistent over time.

In the present study, the RNG task was used to assess changes in selective attention as a function of learning. Subjects were required to master a simple motor skill to the point of overlearning. At selected points during the acquisition, mastery, and overlearning phases of the motor task performance, subjects simultaneously performed the RNG task. ${ }^{1}$ The hypothesis was tested that motor task attentional requirements would be reduced as learning progressed and that such changes would be lawfully reflected in RNG index scores obtained at different points on the motor skill learning curve.

\section{METHOD}

\section{Subjects}

Eight college-age male volunteer subjects performed the motor task for 10 trials, and during Trials 2 and 10 they simultaneously performed the RNG task. This procedure was replicated with six subjects. Three additional subjects performed the motor task for up to 49 trials, with the simultaneous RNG task performed at four points during acquisition and overlearning.

\section{Apparatus}

The motor task of choice was the two-hand coordination test (THC) developed by Melton (1947). The THC testing device (Heinrich Model CM101A) was mounted on a table located at a convenient height directly in front of the subject. Performance of the THC task consisted of manually maintaining contact between the tracker and a small silver target that moved in an irregular path around a black circular background disk at a rate of $1 \mathrm{rpm}$. Tracking was accomplished through the simultaneous rotation of two separate control gears, one moving the tracker forward and backward and the other moving it in the lateral dimension. Each trial required $2 \mathrm{~min}$ and consisted of two complete revolutions of the tracking device. An audible click from the attached timer occurred when contact between tracker and target was lost and again when contact was reestablished. Time on target was assessed by means of a standard timer calibrated to $.01 \mathrm{sec}$. Performance feedback was supplied to the subject by the experimenter after completion of each 2-min THC trial. On trials on which the RNG and THC tasks were performed simultaneously, the numbers produced by the subject were recorded by the experimenter and on audiotape.

\section{Procedure}

Upon entering the experimental room, the subject was seated comfortably, and instructions for the RNG task were administered (see Evans, 1978). A 30-sec practice trial was allowed, and the subject was corrected if he did not keep pace with the metronome or follow instructions. The RNG task was then performed for a 2-min baseline period. After explaining and demonstrating the THC device, the subject then practiced performing the THC task for $1 \mathrm{~min}$.

For the first eight subjects, the tracking task was performed for 102 -min trials interspersed with 15 -sec rest periods, during which performance feedback was provided. During THC Trials 2 and 10 , subjects simultaneously verbalized numbers in random sequence in time with the metronome. Maintaining tracking accuracy on the THC task was stressed. Thus, the THC and RNG tasks were performed together, first when the subjects were essentially unskilled and again after they had the opportunity to acquire skill and competence at the tracking task.

The above procedure was replicated with the groups of six and three subjects. The final three subjects were then required to continue practicing the THC task to the point of complete mastery and then to overlearn the task. Mastery was operationally defined as four consecutive THC trials in which the subject maintained perfect time on target during the entire trial. Overlearning was operationally defined as the performance of an additional 25 trials after mastery had been achieved, together with the subjective report that performance of the task seemed effortless and "automatic." Subjects then performed a THC/RNG simultaneous trial immediately after the initial asymptote of four perfect trials and again after completion of the additional 25 overlearning trials. At the conclusion of these procedures, a final RNG baseline was administered to further assess the effects of practice on RNG index values.

\section{RESULTS}

\section{THC Performance Data}

Analysis of THC time-on-target data for the initial eight subjects showed, as expected, that the rate of motor skill learning was approximately linear over the 10 THC trials $[F(9,81)=27.65, p<.001]$. The linear component accounted for $94 \%$ of the reliable variance if the two combined THC/RNG trials were excluded and $92 \%$ of the variance if the analysis was conducted on all 10 trials. Where nonlinearity occurred, it was due almost exclusively to the rapid improvement observed over the first three trials. By Trials 2 and 10, the efficiency of the THC performance (compared with the subject's own asymptote level of $100 \%$ ) averaged $37 \%$ and $66 \%$, respectively. These findings were replicated for the additional six and three subjects and follow closely the data reported by Melton (1947). Asymptote (four consecutive perfect THC trials) was achieved for the third group between Trials 20 and 25. The THC was then performed by each subject without error for the additional 25 overlearning trials.

\section{RNG Performance Data}

Figure 1 presents mean RNG index scores for baseline and dual-task trials. These RNG data were obtained as a single task during pre- and posttraining baseline assessments and as a dual task at two or four points during acquisition, mastery, and overlearning of the THC task. Mean RNG scores are represented on the ordinate; 


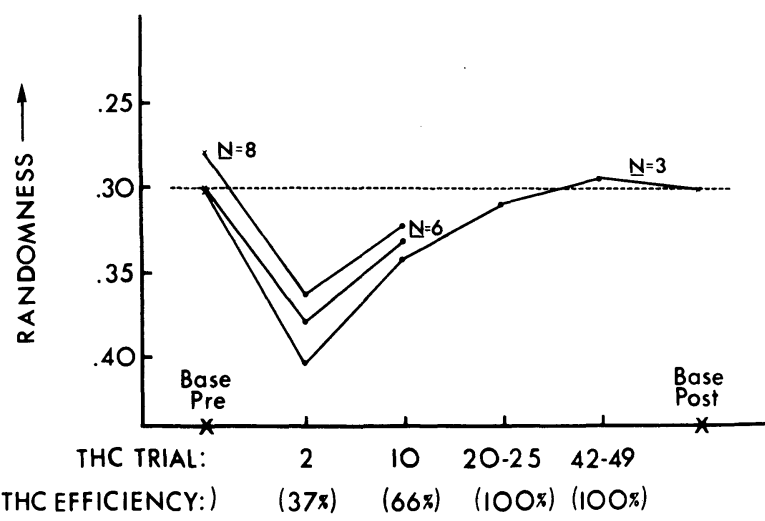

Figure 1. Mean random number generation (RNG) index values obtained during pre- and postbaselines and as a dual task at different levels of skilled performance on the two-hand coordination (THC) task.

more random output is indicated (toward the top) by a smaller index. The number of THC trials is represented on the abscissa, as is the mean percent time subjects remained on target (performance efficiency).

The initial pretraining baseline RNG was .28 $(\mathrm{SD}=.033, \mathrm{~N}=8)$. Performance on RNG deteriorated on combined THC/RNG trials $[\mathrm{F}(2,18)=8.60, \mathrm{p}<.005]$. When the THC task was unlearned and difficult (THC/ RNG combined Trial 2), there was a marked decrement of approximately $2.5 \mathrm{SDs}$ of the original baseline RNG task $[t(7)=3.62, p<.005]$. By Trial 10, when THC performance had improved to $66 \%$ of perfect performance, the ability to simultaneously perform the RNG task had improved considerably $[\mathrm{t}(7)=1.90, \mathrm{p}<.05]$, although Trial $10 \mathrm{RNG}$ was still significantly poorer than the original baseline RNG performance $[t(7)=3.39$, $\mathrm{p}<.02]$. These findings were replicated in both the second $(\mathrm{N}=6)$ and third $(\mathrm{N}=3)$ groups and are shown in Figure 1. When the three subjects had achieved $100 \%$ performance efficiency on the THC task (Trials 20-25), RNG index values were still slightly impaired but were not significantly different from baseline values. After the tracking task was overlearned (Trials 42-49), simultaneous RNG index values also recovered to initial single-task baseline levels. Posttraining RNG baseline values did not differ significantly (for each group) from prebaseline RNG values.

\section{DISCUSSION}

These findings support the original hypothesis that learning reduces initial demands on attention and that such changes are sensitively reflected in RNG index scores obtained at different levels of skilled performance. Compared with baseline RNG performance, randomization ability was severely impaired at low levels of tracking skill, but as subjects began to acquire this skill, they also began to recover their ability to perform the RNG task. When the tracking task was mastered, the ability to simultaneously perform the RNG task had virtually returned to normal levels. During overlearning, when the tracking task had become automatic, randomization ability returned fully to baseline levels.
Practice at the tracking task resulted in a significant performance increment, but practice at the randomization task (from pre- to postbaseline) did not. Although RNG showed no singletrial practice effects, it is possible that it might show practice effects when performed as a simultaneous task. This possibility should be investigated, but we think it is a dubious hypothesis because pilot subjects who performed both tasks simultaneously on each of the first 10 trials had RNG results on Trial 2, Trial 10, and postbaseline that did not differ from those reported above. A hypothesis that deserves further investigation is that the changes observed in simultaneous randomization reflect shifts to a timesharing mode of processing and not alterations in attention capacity. Indeed, Hirst, Spelke, Reaves, Caharack, and Neisser (1980) have postulated skill differences in learning such timesharing, and Jennings and Chiles (1977) have demonstrated individual differences in timesharing abilities involved in complex performance.

Performance of the motor task was assessed on two levels in the present study: acquisition of motor skill as measured by time-on-target values, and task attentional requirements as reflected in RNG index values. Even though the motor skill acquisition curve was virtually linear, the difficulty, or attentional demands, involved in acquiring this skill did not appear to remain constant throughout the learning process. Maximum impairment in simultaneous randomization occurred early in the skill acquisition process but showed significant improvement even when subjects had achieved only $66 \%$ of perfect tracking performance. This suggests that attentional demands are greater during initial acquisition of a skill than during later parts of the learning process, when the early benefits of training are being consolidated. Thus, the amount of attention directed toward a task during acquisition and consolidation phases of learning may not be the same. Attention can be withdrawn and directed to other activities even before the task is mastered.

Many learned skills continue to improve with practice even after mastery is achieved; however, this process has been difficult to document because it is the subjective ease of maintaining perfect performance that seems to change, and not the actual level of performance itself. In other words, a given level of performance effectiveness can be achieved at different levels of performance efficiency, a distinction drawn earlier by Brown (1965). In the present study, given constant (overlearned) performance on the primary task (tracking), an improvement in performance of the index task (randomization) was used to assess alterations in performance efficiency. Thus, when tracking was mastered, randomization ability had not fully returned to baseline levels. With overlearning and the subjective report of automatic, effortless performance, however, simultaneous RNG performance was at the level of single-task RNG baseline values.

The RNG procedure appears useful in assessing attention deployment during the performance of tasks, and, more important, during the learning and acquisition of complex skills. As such, it may provide a brief, sensitive, and consistent measure of attention deployment in a variety of situations in which attention fluctuates during short intervals of time.

\section{REFERENCES}

BADdeley, A. The capacity for generating information by randomization. Quarterly Journal of Experimental Psychology, 1966, 18, 119-129.

Broadbent, D. E. Perception and communication. New York: Pergamon Press, 1958.

Brown, J. S. Generalization and discrimination. In D. L. Mostofsky (Ed.), Stimulus generalization. Stanford: Stanford University Press, 1965.

Evans, F. J. Monitoring attention deployment by random number generation: An index to measure subjective randomness. Bulletin of the Psychonomic Society, 1978, 12, 35-38. 
Graham, C., \& Evans, F. J. Hypnotizability and the deployment of waking attention. Journal of Abnormal Psychology, 1977, 86, 631-638.

Hirst, W., Spelke, E. S., Reaves, C. C., Caharack, G., \& NeISSER, U. Dividing attention without alternation or automaticity. Journal of Experimental Psychology: General, 1980, 109, 98-117.

Jennings, A. E., \& Chiles, W. D. An investigation of timesharing ability as a factor in complex performance. Human Factors, 1977, 19, 535-547.

Kahneman, D. Attention and effort. Englewood Cliffs, N.J: Prentice-Hall, 1973.

LABERgE, D. Acquisition of automatic processing in perceptual and associative learning. In P. M. A. Rabbitt \& S. Dornic (Eds.), Attention and performance $V$. London: Academic Press, 1975.

Melton, A. W. Apparatus tests (Army Air Forces Aviation Psychology Research Rep. 4). Washington, D.C: U.S. Government Printing Office, 1947.

Moray, N. Attention: Selective processes in vision and hearing. London: Hutchinson, 1969.

Noble, M., Trumbo, D., \& Fowler, F. Further evidence on secondary task interference in tracking. Journal of Experimental Psychology, 1967, 73, 146-149.

Treisman, A. Strategies and models of selective attention. Psychological Review, 1969, 76, 282-299.

TUlving, E. Subjective organization in free recall of "unrelated" words. Psychological Review, 1962, 69, 344-354.

WAgenAaR, W. A. Generation of random sequences by human subjects: A critical survey of literature. Psychological Bulletin, 1972, 77, 65-72.

\section{NOTE}

1. Two other studies have used randomization to assess simultaneous performance (Baddeley, 1966; Wagenaar, 1972), but they found opposite effects of simultaneous performance on randomization. These studies were completed when the indexes available to measure randomization were less sensitive than the one used here.

(Received for publication April 15, 1980.) 